\title{
EMPIRICAL DISTRIBUTION FUNCTIONS AND STRONG APPROXIMATION THEOREMS FOR DEPENDENT RANDOM VARIABLES. A PROBLEM OF BAKER IN PROBABILISTIC NUMBER THEORY
}

\author{
WALTER PHILIPP
}

Dedicated to Wolfgang $M$. Schmidt on his 60 th birthday

\begin{abstract}
Let $\mathscr{T}=\left\{q_{1}, \ldots, q_{\tau}\right\}$ be a finite set of coprime integers and let $\left\{n_{1}, n_{2}, \ldots\right\}$ denote the mutiplicative semigroup generated by $\mathscr{T}$, and arranged in increasing order. Let $D_{N}(\omega)$ denote the discrepancy of the sequence $\left\{n_{k} \omega\right\}_{k=1}^{N} \bmod 1, \omega \in[0,1)$. In this paper we solve a problem posed by R.C. Baker [3], by proving that for all $\omega$ except on a set of Lebesgue measure 0

$$
\frac{1}{4} \leq \limsup _{N \rightarrow \infty} \frac{N D_{N}(\omega)}{\sqrt{N \log \log N}} \leq C .
$$

Here the constant $C$ only depends on the total number of primes involved in the prime factorization of $q_{1}, \ldots, q_{\tau}$. The lower bound is obtained from a strong approximation theorem for the partial sums of the sequence $\left\{\cos 2 \pi n_{k} \omega\right\}_{k=1}^{\infty}$ by sums of independent standard normal random variables.
\end{abstract}

\section{INTRODUCTION}

Let $\left\{n_{k}, k \geq 1\right\}$ be an increasing sequence of positive integers and let $([0,1), \mathscr{B}, P)$ denote the unit interval with Lebesgue measurability. Then

$$
\eta_{k}(\omega):=n_{k} \omega \quad \bmod 1
$$

are random variables defined on $([0,1), \mathscr{B}, P)$ which are uniformly distributed in the probabilistic sense, i.e.,

$$
P\left\{\omega: \eta_{k}(\omega) \leq x\right\}=x, \quad 0 \leq x \leq 1, k \geq 1 .
$$

If $F_{N}$ denotes the empirical distribution function of the sequence $\left\{\eta_{k}\right\}$ at stage $N$, i.e.,

$$
F_{N}(s)=F_{N}(s ; \omega):=N^{-1} \#\left\{k \leq N: \eta_{k}(\omega) \leq s\right\}, \quad 0 \leq s \leq 1,
$$

then

$$
D_{N}(\omega):=\sup _{0 \leq s \leq 1}\left|F_{N}(s)-s\right|
$$

denotes the discrepancy (or the Kolmogorov-Smirnov statistic) of the sequence $\left\{n_{k} \omega\right\}_{k=1}^{N} \bmod 1$. (For the basic definition and facts on uniform distribution

Received by the editors October 19, 1992 and, in revised form, January 27, 1993 and October 4, 1993.

1991 Mathematics Subject Classification. Primary 11K38, 11K31; Secondary 60F15. 
$\bmod 1$, see e.g. Kuipers and Niederreiter [13].) Recall that $D_{N}$ is a random variable, i.e., a measurable function from $([0,1), \mathscr{B}, P) \rightarrow([0,1), \mathscr{B}, P)$.

R.C. Baker [2] proved that for given $\varepsilon>0$,

$$
D_{N}(\omega) \ll N^{-\frac{1}{2}}(\log N)^{\frac{3}{2}+\varepsilon}
$$

for almost all $\omega$, i.e., for all $\omega$ except on a set of Lebesgue measure 0 . The best result before Baker's paper had $\frac{5}{2}$ instead of $\frac{3}{2}$ in the exponent of $\log N$ and was independently obtained by Cassels [7] and Erdős and Koksma [10]. This is of course without any further assumptions on $\left\{n_{k}\right\}$.

In 1962 , Erdős $\left[9\right.$, p. 56] conjectured that for some $c\left(\geq \frac{1}{2}\right)$

$$
D_{N}(\omega)=o\left(N^{-\frac{1}{2}}(\log \log N)^{c}\right) \quad \text { a.e. }
$$

Apparently unaware of Erdős' conjecture R.C. Baker [2] conjectured that for any $\varepsilon>0$,

$$
D_{N}(\omega)=o\left(N^{-\frac{1}{2}}(\log N)^{\ell}\right) \quad \text { a.e. }
$$

For exponentially fast growing sequences $\left\{n_{k}\right\}$, i.e., for sequences with

$$
n_{k+1} / n_{k} \geq q>1, \quad k \geq 1,
$$

(1.1) is indeed true. In [19] a bounded law of the iterated logarithm was proved in the form

$$
\frac{1}{4} \leq \limsup _{N \rightarrow \infty} \frac{N D_{N}(\omega)}{\sqrt{N \log \log N}} \leq C(q) \quad \text { a.e. }
$$

Here $C(q) \ll 1 / \log q, q \downarrow 1$, only depends on $q$. This established a longstanding conjecture of Erdős and Gál. By a different method it was shown in [20] that (1.3) continues to hold without the assumption that the $n_{k}$ 's are integers. It is still unknown whether the limes superior equals a constant a.e. Also, the question raised in [19], whether or not the right side (1.3) continues to hold if (1.2) is replaced by

$$
n_{k+1} / n_{k} \geq 1+k^{-q}, \quad q<\frac{1}{2},
$$

still remains open. The left side of (1.3) follows from a result of Berkes [4] and a well-known inequality of Koksma (see [13, p. 143]). $q=\frac{1}{2}$ is the value in (1.4) where the finer probabilistic properties of the partial sums of the sequence $\left\{\cos 2 \pi n_{k} \omega\right\}$ begin to break down (see Berkes [5]). Thus there is some reason to believe that (1.3) is, in general, false for sequences satisfying (1.4) with $q \geq \frac{1}{2}$.

From now on let $\left\{n_{k}\right\}_{k=1}^{\infty}:=\left\{q_{1}^{\alpha_{1}} \ldots q_{\tau}^{\alpha_{\tau}}, \alpha_{i} \geq 0\right.$ integer $\}$ and arranged in increasing order where $\left\{q_{1}, \ldots, q_{\tau}\right\}$ is a finite set of coprime integers. Let $\tau^{*}$ denote the total number of primes occurring in the prime factorization of $q_{1}, \ldots, q_{\tau}$. Let $D_{N}(\omega)$ denote the discrepancy of the sequence $\left\{n_{k} \omega\right\}_{k=1}^{N}$ $\bmod 1$.

The following theorem solves a problem of R.C. Baker [3], posed around 1979. 
Theorem 1. There is a constant $C$, depending on $\tau^{*}$ only such that for almost all $\omega$

$$
\frac{1}{4} \leq \limsup _{N \rightarrow \infty} \frac{N D_{N}(\omega)}{\sqrt{N \log \log N}} \leq C .
$$

Over the years the analytic properties of the sequence $\left\{n_{k} \omega\right\}$ have attracted considerable attention in connection with Khintchine's conjecture. Marstrand [15] proved that for bounded and measurable $f$ with period 1

$$
\frac{1}{N} \sum_{k \leq N} f\left(n_{k} \omega\right) \rightarrow \int_{0}^{1} f(t) d t \quad \text { a.e. }
$$

Recently (1.5) has been proved by Nair [18] to continue to hold under the weakened assumption $f \in L^{1}([0,1))$, thereby answering another question raised by Baker [1].

The lower bound in Theorem 1 will follow from the following strong approximation theorem. Let $\Omega=[0,1)^{2}$, be the unit square with Lebesgue measurability. Write $\omega=\left(\omega_{1}, \omega_{2}\right) \in \Omega$.

Theorem 2. There exists a sequence $\left\{Y_{k}\left(\omega_{1}, \omega_{2}\right)\right\}_{k=1}^{\infty}$ of independent standard Gaussian random variables defined on $[0,1)^{2}$ such that for almost all $\omega=$ $\left(\omega_{1}, \omega_{2}\right) \in[0,1)^{2}$

$$
2^{\frac{1}{2}} \sum_{k \leq N} \cos 2 \pi n_{k} \omega_{1}-\sum_{k \leq N} Y_{k}\left(\omega_{1}, \omega_{2}\right) \ll N^{\frac{1}{2}-\lambda}
$$

for some $\lambda>0$, depending on $\tau^{*}$ only.

By treating $e^{2 \pi i \cdot}=(\cos 2 \pi \cdot, \sin 2 \pi \cdot)$ as a two-dimensional random vector the same proof yields

Theorem 3. There exists a sequence $\left\{Y_{k}\left(\omega_{1}, \omega_{2}\right)\right\}_{k=1}^{\infty}$ of independent standard complex valued Gaussian random variables defined on $[0,1)^{2}$ such that for almost all $\omega=\left(\omega_{1}, \omega_{2}\right) \in[0,1)^{2}$

$$
2^{\frac{1}{2}} \sum_{k \leq N} e^{2 \pi i n_{k} \omega_{1}}-\sum_{k \leq N} Y_{k}\left(\omega_{1}, \omega_{2}\right) \ll N^{\frac{1}{2}-\lambda}
$$

for some $\lambda>0$, depending on $\tau^{*}$ only.

Remark. Since for almost all $\omega \in[0,1)^{2}$

$$
\limsup _{N \rightarrow \infty} \frac{\left|\sum_{k \leq N} Y_{1}\left(\omega_{1}, \omega_{2}\right)\right|}{\sqrt{2 N \log \log N}}=1
$$

by the classical law of the iterated logarithm for 2-dimensional standard Gaussian random vectors, Theorem 3 implies that for almost all $\omega_{1} \in[0,1)$

$$
\limsup _{N \rightarrow \infty} \frac{\left|\sum_{k \leq N} e^{2 \pi i n_{k} \omega_{1}}\right|}{\sqrt{N \log \log N}}=1
$$

The inequality of Koksma with the constant improved by Niederreiter (see [13, p. 143]) implies the lower bound in Theorem 1. The upper bound in Theorem 1 will follow from the following stronger version. 
Theorem 4. Let $\alpha<1 /(4 \tau)$. There is a constant $C$, depending on $\tau^{*}$ only, with the following property. For almost all $\omega \in[0,1)$ there is an $N_{0}=N_{0}(\omega, \alpha)$ such that for all $N \geq N_{0}$ and all $s$ and $t$ with $0 \leq s<t \leq 1$

$$
\max _{k \leq N} k\left|F_{k}(t)-F_{k}(s)-(t-s)\right| \leq C(t-s)^{\alpha}(N \log \log N)^{\frac{1}{2}}+N^{\frac{1}{2}} .
$$

As an immediate consequence we obtain the following corollary. For a proof, see [20, pp. 325-326].

Corollary. Define

$$
f_{N}(t)=N\left(F_{N}(t)-t\right)(2 N \log \log N)^{-\frac{1}{2}}, \quad 0 \leq t \leq 1, N \geq 1 .
$$

Then the sequence $\left\{f_{N}(t), N \geq 1\right\}$ is with probability 1 relatively compact in $D[0,1]$ endowed with the supremum norm.

It follows from a theorem of Tijdeman [24] that the sequence $\left\{n_{k}\right\}$ satisfies a growth condition (1.4) with an effectively computable constant $q>0$. It is easy to see that "on average" (1.4) holds with $q=1-1 / \tau$. Since in general we can assume $\tau \geq 2$ (the case $\tau=1$ is taken care of by (1.2)) it follows by the remarks preceding Theorem 1 that probability methods alone will not suffice for the proof of our results. In fact, the proofs will employ a mix of martingale inequalities, strong approximation theorems for martingales, inequalities from the theory of uniform distribution mod 1 , theorems on the finiteness of the number of solutions of $S$-unit equations, and Tijdeman's theorem.

\section{Preliminary Results}

For fixed $s$ and $t$ with $0 \leq s<t \leq 1$ we write

$$
L=[s, t), \quad x_{\nu}=x_{\nu}(s, t ; \omega)=1_{L}\left(n_{\nu} \omega\right)-(t-s) .
$$

Here $1_{L}(\cdot)$ denotes the indicator function of $L$, extended with period 1 . We also write for any function $f:[0,1)^{2} \rightarrow \mathbf{R}$

$$
\|f\|=\sup _{0 \leq s<t \leq 1}|f(s, t)| \text {. }
$$

Lemma 2.1. Let $\mathscr{N}$ be a finite set of positive integers with $\operatorname{card} \mathscr{N}=N$, say. Then, as $N \rightarrow \infty$

$$
E \sup _{T}\left\|\sum_{\nu \in \mathcal{N}, \nu \leq T} x_{\nu}\right\|^{6} \ll N^{3} \log ^{6} N
$$

where the constant implied by $\ll$ only depends on $\tau^{*}$.

Proof. Note that $\|\cdot\|$ in (2.3) is a random variable since the supremum over $s$ and $t$ needs to be extended only over a countable dense subset of $[0,1)^{2}$.

Let $\mathscr{K} \subset \mathcal{N}$ be an arbitrary subset of $\mathscr{N}$ with card $\mathscr{M}=m$, say. Then by the Erdös-Turán inequality [13, p. 112] we have for each $R \geq 1$ and each $\omega \in[0,1)$

$$
\left\|\sum_{\nu \in \mathcal{R}} x_{\nu}\right\| \leq \frac{6 m}{R}+2 \sum_{r=1}^{R} \frac{1}{r}\left|\sum_{\nu \in \mathcal{R}} e\left(r n_{\nu} \omega\right)\right| .
$$


Here we use the standard notation $e(x)=e^{2 \pi i x}$. Setting $R=m$ we obtain

$$
E\left\{\max _{\not \subset \mathcal{N}}\left\|\sum_{\nu \in \mathcal{M}} x_{\nu}\right\|^{6}\right\} \leq 12^{6}+4^{6} E\left\{\left(\sum_{r \leq N} \frac{1}{r} \max _{R \subset \mathscr{N}}\left|\sum_{\nu \in \mathcal{L}} e\left(r n_{\nu} \cdot\right)\right|\right)^{6}\right\} .
$$

In order to apply one of Billingsley's maximal inequalities [6, p. 102, Problem 5] or better still [17, Theorem 3.1] (see Lemma A.1 in the appendix) we shall show that uniformly in $r=1,2, \ldots$ and uniformly in $M \subset \mathscr{N}$

$$
\int_{0}^{1}\left|\sum_{\nu \in \mathcal{A}} e\left(r n_{\nu} \omega\right)\right|^{6} d \omega \leq A m^{3}
$$

for some constant $A$, depending on $\tau^{*}$ only. The integral in (2.4) is bounded by the number of solutions of the Diophantine equation

$$
\sum_{i=1}^{6} \pm n_{\nu_{i}}=0
$$

with $\nu_{i} \in \mathscr{M}, 1 \leq i \leq 6$. Recall that each $n_{\nu_{i}}$ is a product of at most $\tau^{*}$ prime powers. Hence by the van der Porten-Schlickewei-Evertse theorem (see [22], [11], and [12, pp. 116-117]) there are only finitely many solutions of $(2.5 ; 6)$ with

$$
\text { g.c.d. }\left(n_{\nu_{1}}, n_{\nu_{2}}, n_{\nu_{3}}, n_{\nu_{4}}, n_{\nu_{5}}, n_{\nu_{6}}\right)=1
$$

provided that

$$
\text { no proper subsum of }(2.5 ; 6) \text { vanishes. }
$$

Denote this finite number of solutions of $(2.5 ; 6)$ subject to $(2.6 ; 6)$ and $(2.7)$ by $B_{6}$ and denote the following auxiliary argument $\mathscr{A}_{6}$ :

Let $k \in \mathscr{M}$ and let $\left(n_{\nu_{i}}, 1 \leq i \leq 6\right)$ be one of these $B_{6}$ solutions of $(2.5 ; 6)$. If $n_{k} / n_{\nu_{1}}$ happens to be an integer then the numbers $n_{\nu_{i}} n_{k} / n_{\nu_{1}}(1 \leq i \leq 6)$ could possibly yield a solution of $(2.5 ; 6)$ (provided, of course, that all these six numbers correspond to some $\left.n_{k_{i}}, k_{i} \in \mathscr{M}\right)$. Thus the number of solutions of $(2.5 ; 6)$ subject to $(2.7)$ does not exceed $B_{6} \cdot m$. This is the end of the argument $\mathscr{A}_{6}$.

Since $n_{\nu}>0$ a proper subsum of $(2.5 ; 6)$ can vanish only if either both

$$
n_{\nu_{1}} \pm n_{\nu_{2}} \pm n_{\nu_{3}}=0 \text { and } n_{\nu_{4}} \pm n_{\nu_{3}} \pm \nu_{6}=0
$$

or if both

$$
n_{\nu_{1}} \pm n_{\nu_{2}} \pm n_{\nu_{3}} \pm n_{\nu_{4}}=0
$$

and

$$
n_{\nu_{s}} \pm n_{\nu_{6}}=0 \text {. }
$$

By two arguments $\mathscr{A}_{3}$ (with a self-evident explanation) we see that the number of solutions of $(2.5 ; 6)$ generated by $(2.5 ; 3)$ does not exceed $\left(B_{3} m\right)^{2}$. By an argument $\mathscr{A}_{4}$ we see that the number of solutions of $(2.5 ; 4)$ does not exceed $B_{4} m$ provided that no proper subsum of $(2.5 ; 4)$ vanishes. But this can only happen if both $n_{\nu_{1}}-n_{\nu_{2}}=0$ and $n_{\nu_{3}}-n_{\nu_{4}}=0$ and then the number of solutions 
does not exceed $m^{2}$. Collecting all these estimates and noting that $(2.5 ; 2)$ has at most $m$ solutions we conclude that the total number of solutions of $(2.5 ; 6)$ with $\nu_{i} \in \mathscr{M}, 1 \leq i \leq 6$, does not exceed

$$
6 !\left(m^{3}+B_{3}^{2} m^{2}+B_{4} m^{2}+B_{6} m\right) .
$$

Thus (2.4) holds with $A=6 !\left(1+B_{3}^{2}+B_{4}+B_{6}\right)$. $A^{*}$

Hence by Lemma A.1 with $\alpha=3$, and $\gamma=6$ we obtain for some constant

$$
E \sup _{T}\left|\sum_{\nu \in \mathcal{N}, \nu \leq T} e\left(r n_{\nu} \cdot\right)\right|^{6} \leq A^{*} N^{3} .
$$

Thus by Minkowski's inequality the left-hand side of (2.3) does not exceed

$$
\ll 1+N^{3}\left(\sum_{r \leq N} \frac{1}{r}\right)^{6} \ll N^{3} \log ^{6} N .
$$

Lemma 2.2. Let $\mathscr{N}$ be a finite set of positive integers with card $\mathscr{N}=N$, say, and let $\varepsilon>0$. Then for $0 \leq s<t \leq 1$

$$
E\left(\sum_{\nu \in \mathcal{N}} x_{\nu}(s, t)\right)^{2} \ll(t-s)^{1-\varepsilon} N,
$$

where the constant implied by $\ll$ only depends on $\tau^{*}$ and $\varepsilon$. Moreover,

$$
E\left(\sum_{\nu \in \mathcal{N}} x_{\nu}(s, t)\right)^{4} \ll(t-s)^{\frac{1}{2}(1-\varepsilon)} N^{2}(\log N)^{3} .
$$

Proof. The integral in (2.9) plays an important role in the metric theory of uniform distribution mod 1 . Perhaps the quickest way to prove this lemma is to expand $1_{L}(\cdot)-(t-s)$ into a Fourier series

$$
1_{L}(\omega)-(t-s)=\sum_{h \neq 0} c_{h} e(h \omega)
$$

where

$$
\left|c_{h}\right| \leq \frac{1}{\pi|h|}, \quad h \neq 0
$$

Let $\|\cdot\|_{2}=\left(\int_{0}^{1}|\cdot|^{2} d \omega\right)^{\frac{1}{2}}$ denote the $L^{2}$-norm on $[0,1)$ and let $1\{\cdot, \cdot\}=\delta$.. 
denote the Kronecker symbol. Then by Minkowski's inequality

$$
\begin{aligned}
E^{\frac{1}{2}} & \left\{\left(\sum_{\nu \in \mathcal{N}} x_{\nu}(s, t)\right)^{2}\right\}=\left(\int_{0}^{1}\left(\sum_{\nu \in \mathcal{N}}\left(1_{L}\left(n_{\nu} \omega\right)-(t-s)\right)\right)^{2}\right)^{\frac{1}{2}} \\
& \leq \sum_{u \geq 0}\left\|\sum_{2^{\mu} \leq|h|<2^{u+1}} c_{h} \sum_{\nu \in \mathcal{N}} e\left(h n_{\nu} \cdot\right)\right\|_{2} \\
& =\sum_{u \geq 0}\left(\sum_{\mu, \nu \in \mathcal{N}} \sum_{2^{u} \leq|h|,|k|<2^{\mu+1}} c_{h} \bar{c}_{k} 1\left\{h n_{\mu}, k n_{\nu}\right\}\right)^{\frac{1}{2}} \\
& \leq \sum_{u \geq 0}\left(2 \sum_{\mu \in \mathcal{N}} \sum_{2^{u} \leq|h|<2^{\mu+1}}\left|c_{h}\right|^{2} \sum_{\nu \in \mathcal{N}} \sum_{2^{u} \leq|k|<2^{\mu+1}} 1\left\{h n_{\mu}, k n_{\nu}\right\}\right)^{\frac{1}{2}} .
\end{aligned}
$$

For fixed $u, \mu$, and $h$ the inner sum is easily seen to be $\ll u^{\tau^{*}}$. Indeed, this sum equals the number of solutions of the Diophantine equation $h n_{\mu}=$ $k n_{\nu}$. If $n_{\mu}=p_{1}^{\alpha_{1}} \ldots p_{\tau^{*}}^{\alpha_{q^{*}}}$ and $h=p_{1}^{\gamma_{1}} \ldots p_{\tau^{*}}^{\gamma_{\tau^{*}}} M$, where $M$ is not divisible by $p_{1}, \ldots, p_{\tau^{*}}$ then $k=p_{1}^{\delta_{1}} \ldots p_{\tau^{*}}^{\delta_{r^{*}} M}$ for some nonnegative integers $\delta_{1}, \ldots, \delta_{\tau^{*}}$. But $2^{u} \leq k<2^{u+1}$ implies

$$
p_{1}^{\delta_{1}} \ldots p_{\tau^{*}}^{\delta^{*}} \leq 2^{u+1} \text { or } \delta_{1} \log p_{1}+\cdots+\delta_{\tau^{*}} \log p_{\tau^{*}} \leq(u+1) \log 2 .
$$

The number of lattice points $\left(\delta_{1}, \ldots, \delta_{\tau^{\circ}}\right), \delta_{i} \geq 0$ is about the volume of the corresponding tetrahedron and this is $\ll u^{\tau^{\circ}}$. Thus the number of possible $k$ 's is $\ll \boldsymbol{u}^{\tau^{*}}$. This proves the claim.

Hence we can continue the chain of inequalities and obtain, by (2.12), Cauchy's and Hölder's inequality

$$
\begin{aligned}
& \leq 2 / \pi N^{\frac{1}{2}} \sum_{u \geq 0} u^{\frac{1}{2} \tau^{*}}\left(\sum_{2^{u} \leq h<2^{u+1}}\left|c_{h}\right|^{2}\right)^{\frac{1}{2}} \\
& \ll N^{\frac{1}{2}} \sum_{u \geq 0} u^{\frac{1}{2} \tau^{*}} 2^{-u \varepsilon}\left(\sum_{2^{u} \leq h<2^{u+1}}\left|c_{h}\right|^{2-\varepsilon}\right)^{\frac{1}{2}} \\
& \ll N^{\frac{1}{2}}\left(\sum_{u \geq 0} u^{\tau^{*}} 2^{-2 u \varepsilon}\right)^{\frac{1}{2}}\left(\sum_{u \geq 02^{*} \leq h<2^{u+1}}\left|c_{h}\right|^{2-\varepsilon}\right)^{\frac{1}{2}} \\
& \ll N^{\frac{1}{2}}\left(\sum_{h \neq 0}\left|c_{h}\right|^{2-\varepsilon}\right)^{\frac{1}{2}} \ll N^{\frac{1}{2}}\left(\sum_{h \neq 0}\left|c_{h}\right|^{2-3 \varepsilon} h^{-2 \varepsilon}\right)^{\frac{1}{2}} \\
& \ll N^{\frac{1}{2}}\left(\sum_{h \neq 0}\left|c_{h}\right|^{2}\right)^{\frac{2-\frac{x}{4}}{4}}\left(\sum_{h \neq 0} h^{-2 \varepsilon \varepsilon^{\frac{2}{\varepsilon}}}\right)^{\frac{2}{4}} \\
& \ll N^{\frac{1}{2}}\left\|1_{L}(\cdot)-(t-s)\right\|_{2}^{\frac{1}{2}-\frac{x}{4}} \ll N^{\frac{1}{2}}(t-s)^{\frac{1}{2}-\frac{z}{4}} .
\end{aligned}
$$


This proves (2.9). Relation (2.10) follows by interpolation. Indeed, by [14, p. 156] $\varphi(r):=\log E|X|^{r}$ is a convex function in $r$, for any random variable $X$. Thus by (2.9) and Lemma 2.1

$$
\begin{aligned}
\varphi(4) & =\varphi\left(\frac{1}{2} 2+\frac{1}{2} 6\right) \leq \frac{1}{2}(\varphi(2)+\varphi(6)) \\
& \ll \frac{1}{2}((1-\varepsilon) \log (t-s)+4 \log N+6 \log \log N) .
\end{aligned}
$$

This proves (2.10).

Lemma 2.3. Let $\mathcal{N}$ be as in Lemma 2.2 and let

$$
\varphi_{m}(\omega)=\sum_{|h| \geq m} c_{h} e(h \omega)
$$

where the $c_{h}$ are defined by (2.11). Then for $\lambda>0$

$$
E\left|\sum_{k \in \mathcal{N}} \varphi_{m}\left(n_{k} \omega\right)\right|^{2} \ll N m^{-1}(t-s)^{\frac{1}{t}-\lambda} .
$$

Proof. Let $u_{0}$ be the largest integer with $2^{u_{0}} \leq m$. We follow the proof of Lemma 2.2 until (2.13) and obtain

$$
\begin{aligned}
& E^{\frac{1}{2}}\left\{\left|\sum_{k \in \mathcal{N}} \varphi_{m}\left(n_{k} \omega\right)\right|^{2}\right\} \ll N^{\frac{1}{2}}\left(\sum_{u \geq u_{0}} u^{\tau^{*}} 2^{-2 u \varepsilon}\right)^{\frac{1}{2}}\left(\sum_{u \geq u_{0}} \sum_{2^{*} \leq|h|<2^{m+1}}\left|c_{h}\right|^{2-\varepsilon}\right)^{\frac{1}{2}} \\
& \ll N^{\frac{1}{2}} 2^{-u_{0} \varepsilon} u_{0}^{\tau^{*}}(t-s)^{\frac{1}{2}-\frac{3}{4}}
\end{aligned}
$$

by the remainder of the proof of Lemma 2.2. We let $\varepsilon>\frac{1}{2}$ and obtain the bound

$$
\ll N^{\frac{1}{2}} m^{-\frac{1}{2}}(t-s)^{\frac{1}{t-\lambda}} \quad \text { for } \lambda>0
$$

\section{Proof of Theorem 4}

For $j=0,1,2, \ldots$ we let $H_{j}$ denote the set of all indices $k$ such that

$$
\exp \left(j^{2}\right) \leq n_{k}<\exp \left((j+1)^{2}-j^{\frac{1}{4}}\right)
$$

and we let $I_{j}$ denote the set of all indices $k$ such that

$$
\exp \left((j+1)^{2}-j^{\frac{1}{4}}\right) \leq n_{k}<\exp \left((j+1)^{2}\right) .
$$

Thus if $n_{k}=q_{1}^{\alpha_{1}} \ldots q_{\tau}^{\alpha_{\tau}}$ then $k \in H_{j}$ iff

$$
j^{2} \leq \alpha_{1} \log q_{1}+\cdots+\alpha_{\tau} \log q_{\tau}<(j+1)^{2}-j^{\frac{1}{2}} .
$$

Let $h_{j}$ denote the largest member of $H_{j}$. Estimating the number of lattice points $\left(\alpha_{1}, \ldots, \alpha_{\tau}\right)$ subject to (3.2) we obtain as $j \rightarrow \infty$

$$
h_{j} \sim \frac{1}{\log q_{1} \ldots \log q_{\tau}} \frac{1}{\tau !}\left((j+1)^{2}-j^{\frac{1}{4}}\right)^{\tau} .
$$

and so

$$
\text { card } H_{j} \gg \ll j^{2 \tau-1}, \quad \text { card } I_{j} \ll j^{2 \tau-7 / 4} .
$$

The blocks $I_{j}$ have been introduced to provide the proper spacing between the large blocks $H_{j}$. This is standard technique in the theory of weak dependence. Moreover, the contributions of the blocks $I_{j}$ is negligible. Recall that $\|\cdot\|$ has been defined in (2.2). 
Lemma 3.1. With probability 1 we have as $n \rightarrow \infty$

$$
\left\|\sum_{j \leq n} \sum_{\nu \in I_{j}} x_{\nu}\right\| \ll h_{n}^{\frac{1}{2}-\frac{1}{2 \alpha}} .
$$

Proof. We apply Lemma 2.1 with $\mathscr{N}=\bigcup_{j \leq n} I_{j}$. Then by (3.4)

$$
\begin{aligned}
E\left\|\sum_{j \leq n} \sum_{\nu \in I_{j}} x_{\nu}\right\|^{6} & \ll\left(\sum_{j \leq n} \operatorname{card} I_{j}\right)^{3}\left(\log \left(\sum_{j \leq n} \operatorname{card} I_{j}\right)\right)^{6} \\
& \ll\left(\sum_{j \leq n} j^{2 \tau-7 / 4}\right)^{3}(\log n)^{6} \ll n^{6 \tau-2} .
\end{aligned}
$$

Thus by Markov's inequality we have

$$
P\left(\left\|\sum_{j \leq n} \sum_{\nu \in I_{j}} x_{\nu}\right\| \geq h_{n}^{\frac{1}{2}-\frac{1}{\alpha} \alpha}\right) \ll h_{n}^{-3+\frac{3}{2} \alpha} n^{6 \tau-2} \ll n^{-2+3 \alpha \tau} .
$$

The lemma follows from the convergence part of the Borel Cantelli lemma.

Having properly disposed of the blocks $I_{j}$ we can now concentrate on the blocks $H_{j}$. Let $r_{k}$ denote the largest integer $r$ with

$$
2^{r} \leq n_{k} k^{12}
$$

and let $\mathscr{H}_{k}$ be the $\sigma$-field generated by the intervals

$$
U_{\nu k}=\left[\nu 2^{-r_{k}},(\nu+1) 2^{-r_{k}}\right), \quad 0 \leq \nu<2^{r_{k}} .
$$

We set

$$
\mathscr{F}_{j}=\mathscr{H}_{h_{j}}, \quad \xi_{\nu}=E\left(x_{\nu} \mid \mathscr{F}_{j}\right), \quad \nu \in H_{j}
$$

and

$$
w_{j}=\sum_{\nu \in H_{j}} x_{\nu}, \quad y_{j}=E\left(w_{j} \mid \mathscr{F}_{j}\right)=\sum_{\nu \in H_{j}} \xi_{\nu}
$$

The proof of Theorem 4 follows the pattern of [20, Theorem 4.1] except for the details. We first show that $\left\{y_{j}\right\}$ is close to $\left\{w_{j}\right\}$, uniformly in $0 \leq$ $s<t \leq 1$. Second, we approximate $\left\{y_{j}\right\}$ by a martingale difference $\left\{Y_{j}\right\}$. After truncating $Y_{j}$ and recentering at conditional expectations we apply an exponential inequality. This will finally yield the desired upper bound.

Lemma 3.2. With probability 1

$$
\sum_{j=1}^{\infty}\left\|y_{j}-w_{j}\right\|<\infty
$$

Proof. This will follow from the Beppo Levi theorem and

$$
\sum E\left\|y_{j}-w_{j}\right\|<\infty
$$

In order to show (3.9) we recall that by [20, Lemma 4.2.3] for each $0 \leq s<$ $t \leq 1$

$$
E\left|\xi_{\nu}-x_{\nu}\right|^{2} \ll \nu^{-12}
$$


with an absolute constant implied by $\ll$. We shall use (3.10) to show that in fact

$$
E\left\|\xi_{\nu}-x_{\nu}\right\| \ll \nu^{-3}
$$

and this will yield (3.9) and thus the lemma.

Let $m$ and $M$ be integers with $0 \leq m<M$ which will be chosen suitably later. We write $s$ and $t$ in binary expansion

$$
\begin{array}{ll}
s=\sum_{i=1}^{\infty} \sigma_{i} 2^{-i}, & \sigma_{i}=0,1 \\
t=\sum_{i=1}^{\infty} \tau_{i} 2^{-i}, & \tau_{i}=0,1
\end{array}
$$

Then

$$
\begin{gathered}
s=a 2^{-m}+\sum_{i=m+1}^{M} \sigma_{i} 2^{-i}+\theta_{1} 2^{-M} \\
t=b 2^{-m}+\sum_{i=m+1}^{M} \tau_{i} 2^{-i}+\theta_{2} 2^{-M}
\end{gathered}
$$

where $a$ and $b$ are integers with $0 \leq a, b \leq 2^{m}$ and $0 \leq \theta_{1}, \theta_{2} \leq 1$. We also write for $s<t$

$$
Z=Z(s, t)=Z_{\nu}(s, t)=\left|\xi_{\nu}(s, t)-x_{\nu}(s, t)\right|
$$

and observe that for $s<r<t$

$$
\begin{aligned}
& Z(s, t) \leq Z(s, r)+Z(r, t), \\
& Z(r, t) \leq Z(s, t)+Z(s, r) .
\end{aligned}
$$

We set

$$
m=0, \quad M=\left[\frac{3}{\log 2} \log \nu\right]+1 .
$$

We apply (3.14) repeatedly and obtain in view of (3.12)

$$
\begin{aligned}
Z(s, t) \leq & \sum_{i=1}^{M} Z\left(a_{i} 2^{-i},\left(a_{i}+1\right) 2^{-i}\right)+\sum_{i=1}^{M} Z\left(b_{i} 2^{-i},\left(b_{i}+1\right) 2^{-i}\right) \\
& +Z\left(a_{M+1} 2^{-M},\left(a_{M+1}+1\right) 2^{-M}\right) \\
& +Z\left(b_{M+1} 2^{-M},\left(b_{M+1}+1\right) 2^{-M}\right)+2^{-M+1}
\end{aligned}
$$

where $a_{i}, b_{i}(1 \leq i \leq M+1)$ are integers with $0 \leq a_{i}, b_{i}<2^{i}(1 \leq i \leq M+1)$. The last term is explained by the fact that for $0 \leq h<2^{M}$ and $0 \leq \theta \leq 1$

$$
Z\left(h 2^{-M},(h+\theta) 2^{-M}\right) \leq Z\left(h 2^{-M},(h+1) 2^{-M}\right)+2^{-M}
$$

by an application of (3.14). Thus by (3.10) and (3.15) we obtain

$$
E\|Z\| \leq 4 \sum_{i=1}^{M} \sum_{a=0}^{2^{i}-1} E\left\{Z\left(a 2^{-i},(a+1) 2^{-i}\right)\right\}+2^{-M+1} \leq 2^{M} \nu^{-12} \ll \nu^{-3} \text {. }
$$

This proves (3.11) and thus the lemma. 
Lemma 3.3. The random variables $y_{j}$ can be represented in the form

$$
y_{j}=Y_{j}+v_{j}
$$

where $\left(Y_{j}, \mathscr{F}_{j}, j \geq 1\right)$ is a martingale difference sequence and

$$
v_{j} \ll(t-s) \exp \left(-\frac{1}{2} j^{t}\right) \quad \text { a.s. }
$$

with an absolute constant implied by $\ll$.

Proof. We put $Y_{j}=y_{j}-E\left(y_{j} \mid \mathscr{F}_{j-1}\right)$. By (3.8) $Y_{j}$ is $\mathscr{F}_{j}$-measurable and $E\left(Y_{j} \mid \mathscr{F}_{j-1}\right)=0$. Thus $\left(Y_{j}, \mathscr{F}_{j}, j \geq 1\right)$ is a martingale difference sequence and

$$
v_{j}:=y_{j}-Y_{j}=E\left(y_{j} \mid \mathscr{F}_{j-1}\right)
$$

needs to be estimated. Write $r=r_{h_{j-1}}$ and for $0 \leq k<2^{r}$ let

$$
U=\left[k 2^{-r},(k+1) 2^{-r}\right) \text {. }
$$

By [20, Lemma 4.2.1]

$$
\left|\int_{U} x_{\nu}(\omega) d \omega\right| \leq 4(t-s) n_{\nu}^{-1}
$$

Hence by the definition of a conditional expectation

$$
E\left(x_{\nu} \mid \mathscr{F}_{j-1}\right)=\sum_{k=0}^{2^{r}-1} 1_{U}(\cdot) 2^{r} \int_{U} x_{\nu}(\omega) d \omega \ll 2^{r}(t-s) n_{\nu}^{-1} .
$$

Thus by (3.3), (3.5), and (3.8) we have for $\nu \in H_{j}$

$$
\begin{aligned}
v_{j} & =E\left(y_{j} \mid \mathscr{F}_{j-1}\right)=\sum_{\nu \in H_{j}} E\left(E\left(x_{\nu} \mid \mathscr{F}_{j}\right) \mid \mathscr{F}_{j-1}\right) \\
& =\sum_{\nu \in H_{j}} E\left(x_{\nu} \mid \mathscr{F}_{j-1}\right) \ll n_{h_{j-1}} h_{j-1}^{12} h_{j}(t-s) \exp \left(-j^{2}\right) \\
& \ll(t-s) j^{26 \tau} \exp \left(j^{2}-(j-1)^{\frac{1}{t}}-j^{2}\right) \\
& \ll(t-s) \exp \left(-\frac{1}{2} j^{\frac{1}{4}}\right) \quad \text { a.s. }
\end{aligned}
$$

Lemma 3.4 (Main lemma). Let $C$ be the constant implied by $\ll$ in Lemma 2.2. Then

$$
\sum_{j \leq n} E\left(w_{j}^{2} \mid \mathscr{F}_{j-1}\right) \leq 2 C(t-s)^{\frac{1}{4}-\varepsilon} h_{n}
$$

except on a set of measure $\ll n^{-1 / 32}$.

This will follow from (2.9) and the following two lemmas.

Lemma 3.5. Let $0<\varepsilon<\frac{1}{12}$. Then as $n \rightarrow \infty$

$$
P\left(\max _{k \leq n}\left|\sum_{j \leq k}\left(w_{j}^{2}-E\left(w_{j}^{2} \mid \mathscr{F}_{j-1}\right)\right)\right| \geq(t-s)^{\frac{t}{-\varepsilon} h_{n} n^{-\varepsilon}}\right) \ll n^{-\frac{3}{2}} .
$$

Proof. By (3.10) and Minkowski's inequality

$$
E\left|y_{j}-w_{j}\right|^{2} \leq\left(\sum_{\nu \in H_{j}} E^{\frac{1}{2}}\left|\xi_{\nu}-x_{\nu}\right|^{2}\right)^{2} \ll h_{j}^{-10} .
$$


Thus by (2.9) and since $E y_{j}^{2} \leq E w_{j}^{2}$ by the conditional version of Jensen's inequality

$$
\begin{aligned}
E\left|y_{j}^{2}-w_{j}^{2}\right| & \leq\left(E\left|y_{j}-w_{j}\right|^{2} 2 E w_{j}^{2}\right)^{\frac{1}{2}} \\
& \ll h_{j}^{-5}\left(\operatorname{card} H_{j}\right)^{\frac{1}{2}}(t-s)^{\frac{1}{2-\imath}} \ll(t-s)^{\frac{1}{2}-\imath} j^{-8 \tau}
\end{aligned}
$$

and

$$
E\left|E\left(y_{j}^{2} \mid \mathscr{F}_{j-1}\right)-E\left(w_{j}^{2} \mid \mathscr{F}_{j-1}\right)\right| \leq E\left|y_{j}^{2}-w_{j}^{2}\right| \ll(t-s)^{\frac{1}{2}-\varepsilon} j^{-8 \tau} .
$$

Hence

$$
\begin{aligned}
& P\left(\sum_{j \leq n}\left|y_{j}^{2}-E\left(y_{j}^{2} \mid \mathscr{F}_{j-1}\right)-\left(w_{j}^{2}-E\left(w_{j}^{2} \mid \mathscr{F}_{j-1}\right)\right)\right| \geq(t-s)^{\frac{1}{4}-\varepsilon} h_{n} n^{-\varepsilon}\right) \\
& \quad \ll h_{n}^{-1} n^{\varepsilon} \sum_{j \leq n} j^{-8 \tau} \ll n^{-2 \tau+\varepsilon},
\end{aligned}
$$

and so for the proof of the lemma it is enough to show that

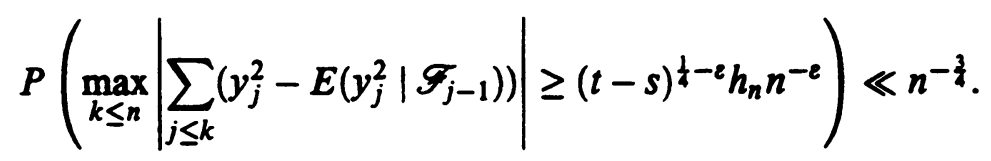

The purpose of this exercise was to make sure that we are dealing with a martingale difference sequence, namely with $\left\{y_{j}^{2}-E\left(y_{j}^{2} \mid \mathscr{F}_{j-1}\right), \mathscr{F}_{j}, j \geq 1\right\}$ which requires $\mathscr{F}_{j}$-measurability of the $j$ th difference. By Doob's maximal inequality for martingales $[8, p .314]$ the probability in question does not exceed

$$
\begin{gathered}
(t-s)^{-\frac{1}{2}+2 \varepsilon} h_{n}^{-2} n^{2 \varepsilon} E\left(\sum_{j \leq n}\left(y_{j}^{2}-E\left(y_{j}^{2} \mid \mathscr{F}_{j-1}\right)\right)\right)^{2} \\
\ll(t-s)^{-\frac{1}{2}+2 \varepsilon} h_{n}^{-2} n^{2 \varepsilon} \sum_{j \leq n} E y_{j}^{4} \\
\ll(t-s)^{-\frac{1}{2}+2 \varepsilon} h_{n}^{-2} n^{2 \varepsilon} \sum_{j \leq n} E w_{j}^{4} \\
\ll h_{n}^{-2} n^{2 \varepsilon} \sum_{j \leq n}\left(\operatorname{card} H_{j}\right)^{2}(\log j)^{3} \ll n^{-1+3 e}
\end{gathered}
$$

using the orthogonality of martingale differences, Jensen's inequality, and (2.10), (3.3), and (3.4).

Lemma 3.6. Let $0<\varepsilon<\frac{1}{32}$. Then as $n \rightarrow \infty$

$$
P\left(\max _{k \leq n}\left|\sum_{j \leq k}\left(w_{j}^{2}-E w_{j}^{2}\right)\right| \geq(t-s)^{\frac{1}{4}-\varepsilon} h_{n} n^{-\varepsilon}\right) \ll n^{-\frac{1}{32}} .
$$

Proof. We set

$$
\begin{gathered}
m_{j}=\left[j^{\mathrm{b}}\right], \\
w_{j}^{*}=\sum_{\nu \in H_{j}} \varphi_{m_{j}}\left(n_{\nu} \omega\right),
\end{gathered}
$$


and

$$
u_{j}=w_{j}-w_{j}^{*}
$$

Then

(3.22) $\left|\left(w_{j}^{2}-E w_{j}^{2}\right)-\left(u_{j}^{2}-E u_{j}^{2}\right)\right| \leq\left|w_{j}^{*}\right|^{2}+E\left|w_{j}^{*}\right|^{2}+2\left|w_{j}^{*} u_{j}\right|+2\left(E\left|w_{j}^{*}\right|^{2} E\left|u_{j}^{*}\right|^{2}\right)^{\frac{1}{2}}$.

Now by Lemma 2.3

$$
E\left|w_{j}^{*}\right|^{2} \ll \operatorname{card} H_{j} m_{j}^{-1}(t-s)^{\frac{1}{4-\varepsilon}} .
$$

Thus by (3.19), (3.3), and (3.4)

$$
\sum_{j \leq n} E\left|w_{j}^{*}\right|^{2} \ll(t-s)^{\frac{1}{t}-\varepsilon} \sum_{j \leq n} j^{-\frac{1}{8}} \operatorname{card} H_{j} \ll(t-s)^{\frac{1}{4}-\varepsilon} n^{-\frac{1}{8}} h_{n} .
$$

Consequently, by Markov's inequality

$$
P\left(\left|\sum_{j \leq n} w_{j}^{*}\right|^{2} \geq(t-s)^{\frac{t}{t-\varepsilon} h_{n} n^{-\varepsilon}}\right) \ll n^{-\frac{1}{16}}
$$

Similarly, since by (2.9)

$$
E\left|u_{j}\right|^{2} \leq 2 E\left|w_{j}\right|^{2}+2 E\left|w_{j}^{*}\right|^{2} \ll \operatorname{card} H_{j}(t-s)^{\frac{1}{4-\varepsilon}}
$$

we have

$$
\begin{aligned}
\sum_{j \leq n}\left(E\left|u_{j}\right|^{2} E\left|w_{j}^{*}\right|^{2}\right)^{\frac{1}{2}} & \ll(t-s)^{\frac{1}{t}-\varepsilon} \sum_{j \leq n} m_{j}^{-\frac{1}{2}} \operatorname{card} H_{j} \\
& \ll(t-s)^{\frac{1}{2-\varepsilon} n^{-\frac{1}{16}} h_{n} .}
\end{aligned}
$$

Consequently

$$
P\left(\sum_{j \leq n}\left|u_{j} w_{j}^{*}\right| \geq(t-s)^{\frac{1}{4}-\varepsilon} h_{n} n^{-\varepsilon}\right) \ll n^{-\frac{1}{32}} .
$$

Thus in order to complete the proof of the lemma we need to estimate

$$
P\left(\max _{k \leq n}\left|\sum_{j \leq n}\left(u_{j}^{2}-E u_{j}^{2}\right)\right| \geq(t-s)^{\frac{1}{2}-\varepsilon} h_{n} n^{-\varepsilon}\right) .
$$

We shall apply Lemma A.2. Let $j<k \leq n$. We need to estimate

$$
E\left|\sum_{j<q \leq k}\left(u_{q}^{2}-E u_{q}^{2}\right)\right|^{2}
$$

Now, by (3.20), (3.21), and (2.11)

$$
\begin{aligned}
& \sum_{j<p \leq q \leq k} E\left(\left(\bar{u}_{p}^{2}-E \bar{u}_{p}^{2}\right)\left(u_{q}^{2}-E u_{q}^{2}\right)\right) \\
& \quad=\sum_{\substack { q \leq k \\
\begin{subarray}{c}{0 \leq\left|h_{1}\right|,\left|h_{2}\right|<m_{q} \\
0 \leq\left|h_{3}\right|,\left|h_{q}\right|<m_{p}{ q \leq k \\
\begin{subarray} { c } { 0 \leq | h _ { 1 } | , | h _ { 2 } | < m _ { q } \\
0 \leq | h _ { 3 } | , | h _ { q } | < m _ { p } } }\end{subarray}} c_{h_{1}} c_{h_{2}} \bar{c}_{h_{3}} \bar{c}_{h_{4}} \cdot \sum_{\nu_{1}, \nu_{2} \in H_{q}} \sum_{j<p \leq q} \sum_{\mu_{1}, \mu_{2} \in H_{p}} I(h, \nu, \mu)
\end{aligned}
$$


where

$$
\begin{aligned}
I(h, \nu, \mu)= & \int_{0}^{1} e\left(\left(h_{1} n_{\nu_{1}}+h_{2} n_{\nu_{2}}-h_{3} n_{\mu_{1}}-h_{4} n_{\mu_{2}}\right) \omega\right) d \omega \\
& -\int_{0}^{1} e\left(\left(h_{1} n_{\nu_{1}}+h_{2} n_{\nu_{2}}\right) \omega\right) d \omega \cdot \int_{0}^{1} e\left(-\left(h_{3} n_{\mu_{1}}+h_{4} n_{\mu_{2}}\right) \omega\right) d \omega
\end{aligned}
$$

Notice that $I(h, \nu, \mu)=0$ or 1 . Consequently, for fixed $h_{1}, h_{2}, h_{3}, h_{4}, \nu_{1}$, and $\nu_{2}$

$$
\sum_{j<p \leq q \leq k} \sum_{\mu_{1}, \mu_{2} \in H_{p}} I(h, \nu, \mu)
$$

is less than the number of solutions of the Diophantine equation

$$
h_{1} n_{\nu_{1}}+h_{2} n_{\nu_{2}}-h_{3} n_{\mu_{1}}-h_{4} n_{\mu_{2}}=0
$$

subject to $h_{1} n_{\nu_{1}} \neq-h_{2} n_{\nu_{2}}$ and $h_{3} n_{\mu_{1}} \neq-h_{4} n_{\mu_{2}}$. According to [12, Corollary 2, p. 129] this number is bounded by $6 \cdot 7^{2 \tau^{\circ}+3} \ll 1$. Indeed, in [12, (5.8)] set $c=-\left(h_{1} n_{\nu_{1}}+h_{2} n_{\nu_{2}}\right), z=1, a=h_{3}$ and $b=h_{4}$. Thus the left-hand side in (3.30) is

$$
\begin{aligned}
& \ll \sum_{j<q \leq k}\left(\operatorname{card} H_{q}\right)^{2} \sum_{\substack{0<\left|h_{i}\right|<m_{q} \\
1 \leq i \leq 4}}\left|c_{h_{1}}\right|^{2}\left|c_{h_{3}}\right|^{2} \\
& \ll(t-s)^{2} \sum_{j<q \leq k} m_{q}^{2}\left(\operatorname{card} H_{q}\right)^{2} .
\end{aligned}
$$

In summary (3.29) is bounded by

$$
(t-s)^{2} \sum_{j<q \leq k} m_{q}^{2}\left(\operatorname{card} H_{q}\right)^{2} \ll(t-s)^{2} \sum_{j<q \leq k} q^{4 \tau-\frac{3}{4}} .
$$

Hence by Lemma A.2 and (3.3) the quantity (3.28) is

$$
\ll h_{n}^{-2} n^{2 \varepsilon} n^{4 \tau-\frac{3}{4}} \ll n^{-\frac{1}{16}} \text {. }
$$

The result follows now from the last estimate combined with (3.22) and (3.24)-(3.28).

Lemma 3.4 follows from Lemmas 3.5 and 3.6 and and $n$-fold application of (2.9).

We now truncate the martingale difference sequence $\left\{Y_{j}, \mathscr{F}_{j}\right\}$ and recenter at conditional expectations. Choose $\rho$ with $\frac{1}{2} \alpha<\rho<1 /(8 \tau)$ and keep it fixed. We define the events

$$
A_{j}=\left\{\left\|Y_{j}\right\| \leq h_{j}^{\frac{1}{2}-\rho}\right\}
$$

and

$$
B_{j}=\left\{\left\|(t-s)^{-\frac{1}{4}+\varepsilon} \sum_{\nu \leq j} E\left(Y_{\nu}^{2} \mid \mathscr{F}_{\nu-1}\right)\right\| \leq 4 C h_{j}\right\}
$$

where $C$ is as in Lemma 3.4. We set

$$
Y_{j}^{*}=Y_{j} 1_{A_{j}} 1_{B_{j}}, \quad W_{j}=Y_{j}^{*}-E\left(Y_{j}^{*} \mid \mathscr{F}_{j-1}\right) \text {. }
$$

Then $\left\{W_{j}, \mathscr{F}_{j}, j \geq 1\right\}$ is a bounded martingale difference sequence close to $\left\{Y_{j}, \mathscr{F}_{j}, j \geq 1\right\}$ as the following two lemmas show. 
Lemma 3.7. With probability 1

Proof. We have by (3.33)-(3.35)

$$
\sum_{j=1}^{\infty}\left\|Y_{j}^{*}-Y_{j}\right\|<\infty
$$

$$
\left\{\left\|Y_{j}^{*}-Y_{j}\right\|>0\right\} \subset A_{j}^{c} \cup B_{j}^{c} .
$$

Now by Lemmas 3.2, 2.1, and (3.33), (3.3), (3.4) and the conditional version of Jensen's inequality

$$
\begin{aligned}
P\left(A_{j}^{c}\right) & \leq P\left(\left\|y_{j}\right\|>\frac{1}{2} h_{j}^{\frac{1}{2}-\rho}\right)+P\left(\left\|v_{j}\right\|>\frac{1}{2} h_{j}^{\frac{1}{2}-\rho}\right) \\
& \ll h_{j}^{-3+6 \rho} E\left\|y_{j}\right\|^{6}+0 \ll h_{j}^{-3+6 \rho} E\left\|E\left(w_{j}^{6} \mid \mathscr{F}_{j}\right)\right\| \\
& \ll h_{j}^{-3+6 \rho} E\left(E\left\|w_{j}\right\|^{6} \mid \mathscr{F}_{j}\right) \ll h_{j}^{-3+6 \rho}\left(\operatorname{card} H_{j}\right)^{3}(\log j)^{6} \\
& \ll j^{-3+12 \rho \tau}(\log j)^{6} .
\end{aligned}
$$

By the convergence part of the Borel Cantelli lemma only finitely many of the events $A_{j}^{c}$ happen with probability 1 .

To show that only finitely many events $B_{j}^{c}$ happen with probability 1 , we argue as follows. For given $j \geq 1$, let $m$ be such that $m^{100} \leq j<(m+1)^{100}$. Since $h_{(m+1)} 100 / h_{m^{100}} \rightarrow 1$ we have for $j \geq j_{0}$

$$
B_{j}^{c} \subset D_{m}:=\left\{\max _{j \leq(m+1)^{100}}\left\|(t-s)^{-t-\varepsilon} \sum_{\nu \leq j} E\left(Y_{\nu}^{2} \mid \mathscr{F}_{\nu-1}\right)\right\|>3 C h_{(m+1)}\right\} .
$$

Hence by Lemmas 3.3, 3.4, and (3.18), $P\left(D_{m}\right) \ll m^{-3}$. Thus with probability 1 only finitely many events $D_{m}$ happen which proves the above claim.

The lemma follows now from (3.36).

Lemma 3.8. As $n \rightarrow \infty$

$$
\sum_{j \leq n}\left\|E\left(Y_{j}^{*} \mid \mathscr{F}_{j-1}\right)\right\|=o\left(h_{n}^{\frac{1}{2}}\right) \quad \text { a.s. }
$$

Proof. Since $E\left(Y_{j} \mid \mathscr{F}_{j-1}\right)=0$ and since $B_{j}$ is $\mathscr{F}_{j-1}$-measurable we have by (3.33), (3.34), and Lemma 3.2

$$
\begin{aligned}
\left\|E\left(Y_{j}^{*} \mid \mathscr{F}_{j-1}\right)\right\| & =\left\|1_{B_{j}} E\left(Y_{j} 1_{A_{j}} \mid \mathscr{F}_{j-1}\right)\right\| \leq\left\|E\left(Y_{j} 1_{A_{j}} \mid \mathscr{F}_{j-1}\right)\right\| \\
& =\left\|E\left(Y_{j} 1_{A_{j}^{c}} \mid \mathscr{Y}_{j-1}\right)\right\| \\
& \ll\left\|E\left(y_{j} 1\left\{\left\|y_{j}\right\| \geq \frac{1}{2} h_{j}^{\frac{1}{2}} j^{-\frac{1}{4}}\right\} \mid \mathscr{F}_{j-1}\right)\right\|+1 \\
& \ll h_{j}^{-\frac{5}{2}+5 \rho}\left\|E\left(y_{j}^{6} \mid \mathscr{F}_{j-1}\right)\right\| .
\end{aligned}
$$

Thus by (3.3) and the estimate for $E\left\|y_{j}\right\|^{6}$ from the proof of Lemma 3.7 we obtain

$$
h_{j}^{-\frac{1}{2}} E\left\|E\left(Y_{j}^{*} \mid \mathscr{F}_{j-1}\right)\right\| \ll j^{-3+10 \rho \tau}(\log j)^{6} .
$$

From the Beppo Levi theorem we conclude that

$$
\sum_{j \geq 1} h_{j}^{-\frac{1}{2}}\left\|E\left(Y_{j}^{*} \mid \mathscr{F}_{j-1}\right)\right\|<\infty \quad \text { a.s. }
$$

The result follows now from the Kronecker lemma.

Finally we can prove the desired exponential bound. 
Lemma 3.9. Let $0<\sigma<2 \rho-\alpha$. Then for all $0 \leq s<t \leq 1$ with $t-s \geq \frac{1}{16} h_{n}^{-\frac{1}{2}}$

$$
P\left(\max _{k \leq n}\left|\sum_{j \leq k} W_{j}\right| \geq 6(t-s)^{\alpha}\left(C h_{n} \log \log h_{n}\right)^{\frac{1}{2}}\right) \ll \exp \left(-4(t-s)^{-\sigma} \log \log h_{n}\right) .
$$

Proof. We apply Lemma A.3 to

$$
d_{j}:= \begin{cases}\frac{1}{2} W_{j} h_{n}^{-\frac{1}{2}}(t-s)^{-\alpha-\frac{1}{2} \sigma} C^{-\frac{1}{2}} & \text { if } j \leq n, \\ 0 & \text { if } j>n\end{cases}
$$

Let

$$
C_{j}=C_{j n}=\left\{\left\|(t-s)^{-t+\varepsilon} \sum_{\nu \leq j} E\left(Y_{\nu}^{2} \mid \mathscr{F}_{\nu-1}\right)\right\| \leq 4 C h_{n}\right\} .
$$

Then $B_{j} \subset C_{j}$ for $j \leq n$ and thus

$$
E\left(W_{j}^{2} \mid \mathscr{F}_{j-1}\right) \leq 1_{B_{j}} E\left(Y_{j}^{2} \mid \mathscr{F}_{j-1}\right) \leq 1_{C_{j}} E\left(Y_{j}^{2} \mid \mathscr{F}_{j-1}\right) .
$$

Consequently, and since $2 \alpha+\sigma<\frac{1}{4}$,

$$
s^{2}=\frac{1}{4} h_{n}^{-1}(t-s)^{-2 \alpha-\sigma} C^{-1} \sum_{j \leq n} E\left(W_{j}^{2} \mid \mathscr{F}_{j-1}\right) \leq 1 .
$$

Moreover,

$$
\left|d_{j}\right| \leq h_{n}^{-\rho}(t-s)^{-\alpha-\frac{1}{2} \sigma} C^{-\frac{1}{2}}=: c .
$$

Thus the probability in question equals

$$
P\left(\sup _{k \leq n}\left|\sum_{j \leq k} d_{j}\right|>3(t-s)^{-\frac{1}{2} \sigma}\left(\log \log h_{n}\right)^{\frac{1}{2}}\right) \ll \exp \left(-4(t-s)^{-\sigma} \log \log h_{n}\right)
$$

since

$$
h_{n}^{-\rho}(t-s)^{-\alpha-\frac{1}{2} \sigma} C^{-\frac{1}{2}} 3(t-s)^{-\frac{1}{2} \sigma}\left(\log \log h_{n}\right)^{\frac{1}{2}} \ll h_{n}^{-\rho}\left(\log \log h_{n}\right)^{\frac{1}{2}} h_{n}^{\frac{1}{2}(\alpha+\sigma)} \rightarrow 0 .
$$

Lemma 3.10. With probability 1 there exists $n_{0}=n_{0}(\omega)$ such that for all $n \geq n_{0}$ and all $s, t$ with $0 \leq s<t \leq 1$

$$
\max _{k \leq n}\left|\sum_{j \leq k} W_{j}\right| \leq 12 \cdot 2^{\tau}(t-s)^{\alpha}\left(C h_{n} \log \log h_{n}\right)^{\frac{1}{2}}+\frac{1}{2} h_{n}^{\frac{1}{2}} .
$$

Proof. As in the proof of Lemma 3.2 we expand $s$ and $t$ in dyadic expansion so that (3.12) holds with $m$ and $M$ chosen suitably below. Instead of (3.13) we define

$$
Z=Z(s, t)=Z(k ; s, t)=\left|\sum_{j \leq k} W_{j}(s, t)\right|
$$


We note that (3.14) still holds. We apply (3.14) repeatedly and obtain instead of (3.16)

$$
\begin{aligned}
Z(s, t) \leq & Z\left(a 2^{-m}, b 2^{-m}\right)+\sum_{i=m+1}^{M} Z\left(a_{i} 2^{-i},\left(a_{i}+1\right) 2^{-i}\right) \\
& +\sum_{i=m+1}^{M} Z\left(b_{i} 2^{-i},\left(b_{i}+1\right) 2^{-i}\right)+Z\left(a_{M+1} 2^{-M},\left(a_{M+1}+1\right) 2^{-M}\right) \\
& +Z\left(b_{M+1} 2^{-M},\left(b_{M+1}+1\right) 2^{-M}\right)+2 h_{n} 2^{-M}
\end{aligned}
$$

where $a, b, a_{i}, b_{i}(m<i \leq M+1)$ are integers with $0 \leq a, b \leq 2^{m}, 0 \leq$ $a_{i}, b_{i}<2^{i}(m<i \leq M+1)$. We set

$$
m=m(n)=\left[\left(\log \log h_{n}\right)^{\frac{1}{2}}\right], \quad M=M(n)=\left[\frac{1}{2 \log 2} \log h_{n}\right]+4
$$

and

$$
\phi(x)=12(C x \log \log x)^{\frac{1}{2}} .
$$

We define the following events:

$$
\begin{aligned}
E_{n}(a, b) & =\left\{\max _{k \leq n} Z\left(h_{k} ; a 2^{-m}, b 2^{-m}\right) \geq\left((b-a) 2^{-m}\right)^{\alpha} \phi\left(h_{n}\right)\right\}, \\
E_{n} & =\bigcup_{0 \leq a, b \leq 2^{m}} E_{n}(a, b), \\
F_{n}(i, a) & =\left\{\max _{k \leq n} Z\left(h_{k} ; a 2^{-i},(a+1) 2^{-i}\right) \geq 2^{-\alpha i} \phi\left(h_{n}\right)\right\}, \\
F_{n} & =\bigcup_{m<i \leq M} \bigcup_{0 \leq a<2^{i}} F_{n}(i, a) .
\end{aligned}
$$

By Lemma 3.9

$$
P\left(E_{n}(a, b)\right) \ll \exp \left(-4 \log \log h_{n}\right) \ll\left(\log h_{n}\right)^{-4}
$$

and thus by (3.38)

$$
P\left(E_{n}\right) \ll 2^{2 m}\left(\log h_{n}\right)^{-4} \ll\left(\log h_{n}\right)^{-3} .
$$

Similarly

$$
P\left(F_{n}(i, a)\right) \ll \exp \left(-4 \cdot 2^{\sigma i} \log \log h_{n}\right)
$$

and so

$$
P\left(F_{n}\right) \ll \sum_{m<i} 2^{i} \exp \left(-4 \cdot 2^{\sigma i} \log \log h_{n}\right) \ll\left(\log h_{n}\right)^{-3}
$$

Consequently by (3.39)

$$
P\left(E_{n} \cup F_{n}\right) \ll\left(\log h_{n}\right)^{-3}
$$

and so

$$
\sum_{p=1}^{\infty} P\left(E_{2 p} \cup F_{2 p}\right)<\infty
$$


The Borel Cantelli lemma implies that with probability 1 only finitely many of the events $E_{2 p}$ or $F_{2 p}$ occur. Let $n$ be sufficiently large and let $p$ be such that $2^{p-1} \leq n<2^{p}$. Then by (3.37) we have with probability 1 for all $0 \leq s<t \leq 1$

$$
\begin{aligned}
\max _{k \leq n} Z(k ; s, t) & \leq\left(\left((b-a) 2^{-m\left(2^{p}\right)}\right)^{\alpha}+2 \sum_{m(2 p) \leq i \leq M(2 p)} 2^{-i \alpha}\right) \phi\left(h_{2 p}\right)+\frac{1}{4} h_{n}^{\frac{1}{2}} \\
& \leq(t-s)^{\alpha} \phi\left(h_{2^{p}}\right)+o\left(h_{2 p}^{\frac{1}{2}}\right)+\frac{1}{4} h_{n}^{\frac{1}{2}} \leq 2^{\tau}(t-s)^{\alpha} \phi\left(h_{n}\right)+\frac{1}{2} h_{n}^{\frac{1}{2}} .
\end{aligned}
$$

This proves the lemma.

Lemma 3.11. Let $\varepsilon<1 /(8 \tau)$. Then with probability 1

$$
\max _{k \leq h_{n}-h_{n-1}}\left\|\sum_{\nu=h_{n-1}+1}^{h_{n-1}+k} x_{\nu}\right\| \ll h_{n}^{\frac{1}{2}-\varepsilon} .
$$

Proof. We apply Lemma 2.1 with $\mathscr{N}=\left(h_{n-1}, h_{n}\right)$ and obtain

$$
\begin{aligned}
& P\left(\max _{k \leq h_{n}-h_{n-1}}\left\|\sum_{\nu=h_{n-1}+1}^{h_{n-1}+k} x_{\nu}\right\| \geq h_{n}^{\frac{1}{2}-\varepsilon}\right) \\
& \quad \leq h_{n}^{-3+6 e}\left(h_{n}-h_{n-1}\right)^{3}(\log n)^{6} \ll n^{-3+12 \varepsilon \tau}(\log n)^{6} .
\end{aligned}
$$

The lemma follows from the convergence part of the Borel Cantelli lemma.

We finally can finish the proof of Theorem 4 . Let $N$ be given. Find $n$ such that $h_{n-1} \leq N<h_{n}$. Then by Lemmas 3.1, 3.2, 3.3, 3.7, 3.8, 3.10, and 3.11 and by (3.3) there exists with probability 1 and $N_{0}=N_{0}(\alpha, \omega)$ such that for all $N \geq N_{0}$ and all $s, t$ with $0 \leq s<t \leq 1$

$$
\begin{aligned}
& \max _{k \leq N} k\left|F_{k}(t)-F_{k}(s)\right| \leq \max _{p \leq n} h_{p}\left|F_{h_{p}}(t)-F_{h_{p}}(s)\right|+\max _{q \leq h_{n}-h_{n-1}}\left\|\sum_{\nu=h_{n-1}+1}^{h_{n-1}+q} x_{\nu}\right\| \\
& \quad \leq 12 \cdot 2^{\tau}(t-s)^{\alpha}\left(C h_{n} \log \log h_{n}\right)^{\frac{1}{2}}+\frac{1}{2} h_{n-1}^{\frac{1}{2}}+o\left(h_{n}^{\frac{1}{2}}\right) \\
& \quad \leq 13 \cdot 2^{\tau}(t-s)^{\alpha}(C N \log \log N)^{\frac{1}{2}}+N^{\frac{1}{2}} .
\end{aligned}
$$

\section{Proof of Theorem 2}

We shall apply Lemma A.4. The random variable

$$
U(\omega)=U\left(\omega_{1}, \omega_{2}\right)=\omega_{2}, \quad \omega \in[0,1)^{2},
$$

has uniform distribution and is independent of the sequence $\left\{\cos 2 \pi n_{\nu} \omega_{1}\right.$, $\nu \geq 1\}$.

Let $H_{j}, I_{j}$ and $\mathscr{F}_{j}$ be defined as in Section 3. let $\mathscr{F}_{j}^{\prime}$ be the set of all rectangles $A \times[0,1)$ where $A \in \mathscr{F}_{j}$. We define

$$
\xi_{\nu}=\xi_{\nu}\left(\omega_{1}, \omega_{2}\right)=2^{\frac{1}{2}} E\left(\cos 2 \pi n_{\nu} \cdot \mid \mathscr{F}_{j}^{\prime}\right), \quad \nu \in H_{j} .
$$

Then $\xi_{\nu}$ is a random variable defined on $[0,1)^{2}$ by

$$
\xi_{\nu}\left(\omega_{1}, \omega_{2}\right)=2^{r_{h_{j}}+\frac{1}{2}} \int_{U_{k h_{j}}} \cos 2 \pi n_{\nu} u d u, \quad \omega_{1} \in U_{k h_{j}}, \quad \omega_{2} \in[0,1),
$$


for $\nu \in H_{j}$. Thus by (3.5) we have for all $\left(\omega_{1}, \omega_{2}\right) \in[0,1)^{2}$

$$
\left|\xi_{\nu}\left(\omega_{1}, \omega_{2}\right)-2^{\frac{1}{2}} \cos 2 \pi n_{\nu} \omega_{1}\right| \leq 4 \pi n_{\nu} 2^{-r_{h_{j}}} \ll h_{j}^{-12}, \quad \nu \in H_{j} .
$$

We set

$$
y_{j}:=\sum_{\nu \in H_{j}} \xi_{\nu}
$$

and

$$
X_{j}:=y_{j}-E\left(y_{j} \mid \mathscr{F}_{j-1}^{\prime}\right) \text {. }
$$

Then $\left\{X_{j}, F_{j}^{\prime}, j \geq 1\right\}$ is a martingale difference sequence and this is the sequence to which we later apply Lemma A.4. We first show that it is an adequate approximation for the partial sums of the cosine series.

Lemma 4.1. For almost all $\left(\omega_{1}, \omega_{2}\right) \in[0,1)^{2}$

$$
\sum_{\nu \leq h_{n}} 2^{\frac{1}{2}} \cos 2 \pi n_{\nu} \omega_{1}-\sum_{j \leq n} X_{j} \ll h_{n}^{\frac{1}{2}(1-\alpha)}
$$

where $\alpha<1 /(4 \tau)$ is as in Theorem 4 and Section 3.

Proof. By (4.1)

$$
\sum_{\nu=1}^{\infty}\left|\xi_{\nu}-2^{\frac{1}{2}} \cos 2 \pi n_{\nu} \cdot\right|<\infty
$$

Since for $0 \leq a<b \leq 1$

$$
\left|\int_{a}^{b} \cos 2 \pi n_{\nu} u d u\right| \leq 2 n_{\nu}^{-1}
$$

we have for $\nu \in H_{j}$

(4.5) $\left|E\left(\xi_{\nu} \mid \mathscr{F}_{j-1}^{\prime}\right)\right|=\left|E\left(\cos 2 \pi n_{\nu} \cdot \mid \mathscr{F}_{j-1}^{\prime}\right)\right|^{\frac{1}{2}} \leq 2^{r_{h_{j-1}}+\frac{3}{2}} n_{\nu}^{-1} \ll \exp \left(-\frac{1}{2} j^{\frac{1}{4}}\right)$

by (3.5) and (3.1). Hence by (3.3)

$$
\sum_{j=1}^{\infty}\left|X_{j}-y_{j}\right| \ll \sum_{j=1}^{\infty} h_{j} \exp \left(-\frac{1}{2} j^{\frac{1}{4}}\right)<\infty .
$$

Finally by Koksma's inequality and Lemma 3.1 we have with probability 1

$$
\left\|\sum_{j \leq n} \sum_{\nu \in I_{j}} \cos 2 \pi n_{\nu} \cdot\right\| \ll h_{n}^{\frac{1}{2}(1-\alpha)} .
$$

This with (4.4) and (4.6) proves the lemma.

Lemma 4.2. For some $\rho>0$, depending on $\tau$ only

$$
V_{n}:=\sum_{j \leq n} E\left(X_{j}^{2} \mid \mathscr{F}_{j-1}^{\prime}\right)=h_{n}+O\left(h_{n}^{1-\rho}\right) \quad \text { a.s. }
$$

where the constant implied by $O$ is nonrandom. 
Proof. By (4.3), (4.5), and (4.1),

$$
\begin{aligned}
E\left(X_{j}^{2} \mid \mathscr{F}_{j-1}^{\prime}\right) & =E\left(y_{j}^{2} \mid \mathscr{F}_{j-1}^{\prime}\right)+O\left(h_{j} \exp \left(-\frac{1}{2} j^{\frac{1}{4}}\right)\right) \\
& =\sum_{\mu, \nu \in H_{j}} E\left(\xi_{\mu} \xi_{\nu} \mid \mathscr{F}_{j-1}^{\prime}\right)+O\left(\exp \left(-\frac{1}{4} j^{\frac{1}{4}}\right)\right) \\
& =2 \sum_{\mu, \nu \in H_{j}} E\left(\cos 2 \pi n_{\mu} \cdot \cos 2 \pi n_{\nu} \cdot \mid \mathscr{F}_{j-1}^{\prime}\right)+O\left(h_{j}^{-10}\right) \\
& =\operatorname{card} H_{j}+O\left(h_{j}^{-10}\right) .
\end{aligned}
$$

The last relation comes from the fact that on an interval $U$ of the form (3.17) a typical conditional expectation equals

$$
2^{r_{k}} \cdot \frac{1}{2} \int_{U} \cos \left(2 \pi\left(n_{\mu}+n_{\nu}\right) u\right) d u+2^{r_{k}} \cdot \frac{1}{2} \int_{U} \cos \left(2 \pi\left(n_{\mu}-n_{\nu}\right) u\right) d u .
$$

Thus for $\mu=\nu$ the second term equals $\frac{1}{2}$ and this explains the main term. Because of (3.3), (3.1), and (3.5) and because of Tijdeman's theorem [24] in all other cases the above terms are bounded by

$$
\begin{aligned}
2^{r_{k}}\left|n_{\nu}-n_{\mu}\right|^{-1} & \leq 2^{r_{k}} \max _{i \in H_{j}} \frac{\left(\log n_{i}\right)^{C_{1}}}{n_{i}} \\
& \ll \exp \left(j^{2}-(j-1)^{\frac{1}{4}}\right) h_{j-1}^{12} \cdot e^{-j^{2}} j^{2 C_{1}} \ll \exp \left(-\frac{1}{4} j^{\frac{1}{4}}\right) .
\end{aligned}
$$

Thus

$$
\begin{aligned}
V_{n} & =\sum_{j \leq n} \operatorname{card} H_{j}+O(1) \\
& =h_{n}-\sum_{j \leq n} \operatorname{card} I_{j}+O(1)=h_{n}+O\left(h_{n}^{1-\rho}\right) .
\end{aligned}
$$

We now apply Lemma A.4 with $f(x)=x^{1-\varepsilon}, \varepsilon<1 /(4 \tau)$. Using (2.8) and (3.3) we see that a typical term in (A3) is bounded by

$$
V_{n}^{-3+3 e} E\left|X_{n}\right|^{6} \ll h_{n}^{-3+3 e}\left(\text { card } H_{n}\right)^{3} \ll n^{-3+6 e r} \text {. }
$$

Thus (A3) holds. Hence we obtain a sequence $\left\{Y_{j}\left(\omega_{1}, \omega_{2}\right), j \geq 1\right\}$ of independent standard normal variables such that with probability 1

$$
\sum_{n \geq 1 ; h_{n}+O\left(h_{n}^{1-\rho}\right) \leq t} X_{n}-\sum_{j \leq t} Y_{j} \ll t^{\frac{1}{2}-\varepsilon / 50} .
$$

In view of Lemma 4.1 this implies

$$
2^{\frac{1}{2}} \sum_{\nu \leq h_{n}} \cos 2 \pi n_{\nu} \omega_{1}-\sum_{j \leq h_{n}+O\left(h_{n}^{1-\rho}\right)} Y_{j}\left(\omega_{1}, \omega_{2}\right) \ll h_{n}^{\frac{1}{2}-\varepsilon / 50} .
$$

Koksma's inequality [13, p. 143] and Lemma 3.11 imply

$$
\max _{k \leq h_{n}-h_{n-1}}\left|\sum_{\nu=h_{n-1}+1}^{h_{n-1}+k} \cos 2 \pi n_{\nu} \omega_{1}\right| \ll h_{n}^{\frac{1}{2}-\varepsilon} \quad \text { a.s. }
$$


and Levy's maximal inequality implies that

$$
\max _{k \leq h_{n}-h_{n-1}+O\left(h_{n}^{1-\rho}\right)}\left|\sum_{\nu=h_{n-1}+1}^{h_{n-1}+k} Y_{j}\left(\omega_{1}, \omega_{2}\right)\right| \ll h_{n}^{\frac{1}{2}-\lambda} \quad \text { a.s. }
$$

for some $\lambda>0$, depending only on $\rho$. Theorem 2 follows from (4.7), (4.8), and (4.9).

\section{APPENDIX}

Let $g(i, j)$ be a superadditive function, i.e., a function satisfying

$$
\begin{gathered}
g(i, j) \geq 0 \quad \text { for all } 1 \leq i \leq j \leq n \\
g(i, j) \leq g(i, j+1) \quad \text { for all } 1 \leq i \leq j \leq n \\
g(i, j)+g(j+1, k) \leq \boldsymbol{g}(i, k) \quad \text { for all } 1 \leq i \leq j \leq k \leq n
\end{gathered}
$$

The following lemma is a special case of [17, Theorem 3.1].

Lemma A.1. Let $\alpha>1$ and $\gamma \geq 1$ be given. Let $X_{1}, X_{2}, \ldots$ be a sequence of random variables with finite $\gamma$ th moments. Suppose that there exists a superadditive function $g(i, j)$ such that

$$
E\left|\sum_{i \leq \nu \leq j} x_{\nu}\right|^{\gamma} \leq g^{\alpha}(i, j) \quad \text { for all } 1 \leq i \leq j \leq n .
$$

Then there exists a constant $A$, depending only on $\alpha$ and $\gamma$ (but not on $n, X_{k}$, or otherwise on $g$ ) such that

$$
E \max _{k \leq n}\left|\sum_{\nu \leq k} X_{\nu}\right|^{\nu} \leq A g^{\alpha}(1, n) .
$$

There are similar results when (A1) is replaced by probability estimates [6, Theorem 12.2] and [17, Theorem 3.2]. A result of this type is needed in the proof of Lemma 3.6. Unfortunately, none of the two above results is easy to apply in this situation. The following lemma is not as sharp, but it applies easily and is almost trivial.

Lemma A.2. Let $X_{1}, X_{2}, \ldots$ be a sequence of random variables with $k$ th partial sum $S_{k}$. Let $n \geq 1$ and $t \geq 0$ be fixed. Suppose that for some $u_{\nu} \geq 0,1 \leq$ $\nu \leq n$,

$$
P\left(\left|S_{k}-S_{j}\right| \geq t\right) \leq \sum_{\nu=j+1}^{k} u_{\nu} \quad \text { for all } 1 \leq j<k \leq n \text {. }
$$

Then

$$
P\left(\max _{k \leq n}\left|S_{k}\right| \geq 2 t \log n\right) \leq \sum_{\nu \leq n} u_{\nu}
$$

Proof. Write $k \leq n$ in dyadic expansion

$$
k=2^{p}+\epsilon_{1} 2^{p-1}+\cdots+\epsilon_{p}
$$


where $\epsilon_{i}=0,1(1 \leq i \leq p)$. Then $p<2 \log k \leq 2 \log n$ and

$$
\left|S_{k}\right| \leq\left|S_{2 p}\right|+\left|S_{2 p+2 p-1}-S_{2 p}\right|+\ldots .
$$

Thus applying (A2) $p$ times we obtain the result.

We quote a special case of a recent result of Pinelis [21].

Lemma A.3. Let $\left\{f_{n}, \mathscr{F}_{n}, n \geq 0\right\}$ be a real-valued martingale $f_{0} \equiv 0$, and let $d_{n}=f_{n}-f_{n-1}, n=1,2, \ldots, d_{0} \equiv 0$ be its associated martingale difference sequence. Put

$$
\begin{aligned}
& f^{*}=\sup \left\{\left|f_{n}\right|, n=0,1,2, \ldots\right\}, \\
& d^{*}=\sup \left\{\left|d_{n}\right|, n=0,1,2, \ldots\right\},
\end{aligned}
$$

and

$$
s=\left(\sum_{n=1}^{\infty} E\left(d_{n}^{2} \mid \mathscr{I}_{n-1}\right)\right)^{\frac{1}{2}} .
$$

Suppose that ess sup $s \leq 1$ and ess sup $d^{*} \leq c$, for some $c>0$. Then

$$
P\left(f^{*}>r\right)<2 \exp \left(\frac{r}{c}-\left(\frac{r}{c}+\frac{2}{c^{2}}\right) \log \left(1+\frac{r c}{2}\right)\right), \quad r \geq 0 .
$$

We also need a theorem of Strassen [23] which we restate in a form more convenient for our purposes (see [16, Theorem 7]).

Lemma A.4. Let $\left\{X_{n}, \mathscr{F}_{n}, n \geq 1\right\}$ be a real-valued square-integrable martingale difference sequence, defined on some probability space $(\Omega, \mathscr{F}, P)$. Let $f$ be a nondecreasing function with $f(x) \rightarrow \infty$ as $x \rightarrow \infty$ and such that $\frac{f(x)(\log x)^{\alpha}}{x}$ is nonincreasing for some $\alpha>50$. Suppose that

$$
V_{n}:=\sum_{j \leq n} E\left(X_{j}^{2} \mid \mathscr{F}_{j-1}\right) \rightarrow \infty \quad \text { a.s. }
$$

and that

$$
\sum_{n \geq 1} E\left\{X_{n}^{2} 1\left\{X_{n}^{2}>f\left(V_{n}\right)\right\} / f\left(V_{n}\right)\right\}<\infty .
$$

Suppose there exists a random variable $U$, uniformly distributed over $[0,1)$ and independent of the sequence $\left\{X_{n}\right\}$. Then there exists a sequence $\left\{Y_{n}, n \geq 1\right\}$ of independent standard normal random variables defined on $(\Omega, F, P)$ such that with probability 1

$$
\sum_{n \geq 1} x_{n} 1\left\{V_{n} \leq t\right\}-\sum_{m \leq t} Y_{m} \ll t^{\frac{1}{2}}(f(t) / t)^{1 / 50}
$$

Acknowledgment. I would like to thank István Berkes for his comments on the original draft of this paper.

Note added in September 1993.

In a recent paper, I. Berkes and W. Philipp, The size of trigonometric and Walsh series and uniform distribution mod 1, J. London Math. Soc. (to appear), a counterexample to the Erdös-Baker conjecture (1.1) is provided. In addition, we prove that for sequences $\left\{n_{k}\right\}$ satisfying (1.4) the upper half of the law of the iterated logarithm (1.3) is indeed false. 


\section{REFERENCES}

1. R. C. Baker, R.C., Riemann sums and Lebesgue integrals, Quart. J. Math. Oxford Ser. 227 (1976), 191-198.

2. __ Metric number theory and the large sieve, J. London Math. Soc. (2) 24 (1981), 34-40.

3. _ Personal communication, 1979.

4. I. Berkes, An almost sure invariance principle for lacunary trigonometric series, Acta Math. Acad. Sci. Hungar. 26 (1975), 209-220.

5. and Statistics (Pécz, 1989), Colloq. Math. Soc. János Bolyai 57, North-Holland, 1991, pp. 35-58.

6. P. Billingsley, Convergence of probability measures, Wiley, New York, 1968.

7. J. W. S. Cassels, Some metrical theorems in Diophantine approximation. III, Proc. Cambridge Philos. Soc. 46 (1950), 219-225.

8. J. L. Doob, Stochastic processes, Wiley, New York, 1953.

9. P. Erdös, Problems and results on diophantine approximations, Compositio Math. 16 (1964), 52-69.

10. P. Erdös and J. F. Koksma, On the uniform distribution modulo one of sequence $(f(n, \theta))$, Proc. Kon. Nederl. Akad. Wetensch. 52 (1949), 851-854.

11. J.-H. Evertse, On sums of $S$-units and linear recurrences, Compositio Math. 53 (1984), 225-244.

12. J.-H. Evertse, K. Györy, C. L. Stewart, and R. Tijdeman, S-unit equations and their applications, New Advances in Transcendence Theory (A. Baker, ed.), Cambridge Univ. Press, London and New York, 1988, pp. 110-174.

13. L. Kuipers and H. Niederreiter, Uniform distribution of sequence, Wiley, New York, 1974.

14. M. Loève, Probability theory, 3rd ed., Van Nostrand, Princeton, NJ, 1963.

15. J. M. Marstrand, On Khinchin's conjecture about strong uniform distribution, Proc. London Math. Soc. (3) 21 (1970), 540-556.

16. D. Monrad and W. Philipp, Nearby variables with nearby conditional laws and a strong approximation theorem for Hilbert space valued martingales, Probab. Theory Related Fields 88 (1991), 381-404.

17. F. A. Moricz, R.J. Serfling, and W.F. Stout, Moment and probability bounds with quasisuperadditive structure for the maximum partial sum, Ann. Probab. 10 (1982), 1032-1040.

18. R. Nair, On strong uniform distribution, Acta Arith. 56 (1990), 183-193.

19. W. Philipp, Limit theorems for lacunary series and uniform distribution mod 1, Acta Arith. 26 (1975), 241-251.

20. dependent random variables, Ann. Probab. 5 (1977), 319-350.

21. I. F. Pinelis, An approach to inequalities for the distributions of infinite-dimensional martingales, Probability in Banach Spaces, Proc. Eighth Internat. Conf., Birkhäuser, 1992.

22. A. J. van der Porten and H.P. Schlickewei, The growth conditions for recurrence sequences, Macquarie Univ. Math. Rep., 82-0041, North Ridge, Australia, 1982.

23. V. Strassen, Almost sure behavior of sums of independent random variables and martingales, Proc. Fifth Berkeley Sympos. Math. Stat. Probab., Vol. II, Part I, Univ. of California Press, 1967, pp. 315-343.

24. R. Tijdeman, On integers with many small prime factors, Compositio Math. 26 (1973), 319-330.

Department of Statistics, University of Illinois, 725 S. Wright Street, Champaign, ILINOIS 61820 